\title{
Upper limb asymmetries in the utilization of proprioceptive feedback
}

Received: 1 August 2005/ Accepted: 22 October 2005 / Published online: 25 November 2005

(C) Springer-Verlag 2005

\begin{abstract}
Despite the importance of proprioception during upper limb movement, the extent to which arm/ hemisphere asymmetries exist in the utilization of proprioceptive feedback remains unclear. In the present study, movement accuracy and arm dynamics were examined in 20 right-handed adults during a proprioceptive matching task that required subjects to actively match remembered target positions of the elbow with the contralateral arm. As hypothesized, the results indicated an accuracy advantage in favor of the nonpreferred left arm reflected by smaller absolute matching errors when compared to the preferred right arm. This advantage was most pronounced for larger amplitude movements and was not associated with any limb-specific difference in movement strategy as indicated by the dynamics of the matching movement. These results extend current theories of handedness by demonstrating that, in right-handed individuals, the non-preferred arm/ hemisphere system is more adept at utilizing positionrelated proprioceptive information than the preferred $\mathrm{arm} /$ hemisphere system.
\end{abstract}

Keywords Handedness - Kinematics · Hemispheric specialization $\cdot$ Human movement

\section{Introduction}

Handedness can be defined as the preferential use of one arm versus the other for motor activities and has been regarded as the most obvious example of lateralized brain function in humans. As such, the extent to which handedness is associated with an asymmetry in motor performance has been the focus of numerous research

D. J. Goble · C. A. Lewis · S. H. Brown $(\bowtie)$

Motor Control Laboratory, Division of Kinesiology, University of Michigan, 401 Washtenaw Ave., Ann Arbor, MI 48109-2214, USA E-mail: shcb@umich.edu

Tel.: + 1-734-7635829

Fax: + 1-763-9361925 investigations. For example, the preferred right arm of healthy adults has been shown to be faster (Flowers 1975; Elliott et al. 1999), more accurate (Carson et al. 1993; Roy et al. 1994) and less variable (Elliott et al. 1986, 1999) than the non-preferred left arm. This bias in motor performance in favor of the preferred arm (and presumably contralateral hemisphere) has given rise to the term 'dominant arm' or, more specifically, the 'dominant arm/hemisphere system'.

The neurophysiological basis of handedness has been debated with two complementary theories emerging over the past several decades. In the open versus closed loop hypothesis of handedness, arm differences have been attributed to feedforward advantages in the planning of a motor response and/or feedback-based advantages in the utilization of sensory information to correct ongoing movement (Flowers 1975; Annett et al. 1979; Haaland and Harrington 1989a, b; Carson et al. 1993; Winstein and Pohl 1995; Mieschke et al. 2001). In the dynamic dominance hypothesis of handedness, the preferred arm/ hemisphere system is thought to be specialized for limb trajectory control, whereas the non-preferred arm/ hemisphere system is specialized for obtaining static limb positions or postures (Bagesteiro and Sainburg 2003; Sainburg 2002; Wang and Sainburg 2003, 2004).

An important consideration in any theory of motor control is the ability to generate and utilize sensory information. Indeed, sensory feedback plays a significant role in all aspects of movement including updating motor plans based on previous movement experience and monitoring movement execution through comparisons of predicted and actual movement outcomes. Although vision and proprioception provide primary sources of sensory information during purposive movement, most studies of handedness have been conducted under conditions of visual guidance where an assessment of feedback utilization may be biased toward visual rather than proprioceptive information. This is despite the important role of proprioceptive feedback during movement, including the control of muscle interaction torques (Sainburg et al. 1995), limb segment timing 
(Cordo et al. 1994) and acquisition of skilled movement acquisition (Kawato 1999).

To what extent asymmetries exist in the utilization of proprioceptive feedback remains unclear. Roy and MacKenzie (1978) examined the accuracy of subjects when matching thumb and multi-joint arm positions in the absence of vision and found a non-preferred left limb advantage for matching thumb position, while no limb differences were evident for arm matching. Using a similar paradigm, these observations were expanded upon by Colley (1984), who found evidence of improved matching with the left thumb, and Carnahan and Elliott (1987), who demonstrated left leg dominance for reproducing target positions with the feet. These results are contrasted, however, by the more recent reports of Chapman et al. (2001) and Carson et al. (1990) who failed to provide evidence of limb asymmetry for matching target end-point in either two or three dimensions, respectively.

The present study examined asymmetries in the utilization of proprioceptive feedback during a task that required subjects to actively match remembered target positions of the elbow with the contralateral arm. Based on current theories of handedness that suggest a specialization of the non-preferred limb/hemisphere system for utilizing sensory feedback and/or obtaining limb postures, it was hypothesized that matching performance would be enhanced in the non-preferred compared to the preferred arm.

\section{Methods}

\section{Subjects}

Twenty healthy adults (mean age $20.8 \pm 2.1$ years; ten males, ten females) recruited from the university community participated in the study. Subjects were strongly right handed as measured by a ten-item version of the Edinburgh inventory (Oldfield 1971) and gave informed consent prior to testing. All experiments followed the ethical guidelines specified by the Institutional Review Board of the University of Michigan.

\section{Experimental set-up and design}

Subjects were blindfolded and seated at an adjustable table with their forearms resting comfortably on two instrumented manipulanda designed specifically for measuring elbow angular displacement in the horizontal plane. Each manipulandum consisted of an adjustable metal support mounted on a frictionless pivot beneath the elbow joint. Subjects grasped a vertical handle at the distal end of the support. Standardized start positions for the shoulder $\left(60^{\circ}\right.$ abduction, $20^{\circ}$ flexion), elbow $\left(115^{\circ}\right.$ extension) and wrist (neutral) were maintained across subjects.
During the matching task, the subject's forearm was passively displaced by the experimenter to one of the two target positions $\left(20^{\circ}, 40^{\circ}\right.$ elbow extension from start position), held at the target position for $2 \mathrm{~s}$ and then returned to the start position. Active matching of the target position was then performed using the contralateral arm with an emphasis placed on end-point accuracy. This procedure was chosen based on its relative difficulty, as evidenced by studies showing greater matching error with increased target distance (Lonn et al. 2000) and in tasks requiring the generation and interhemispheric transfer of a remembered target location (Goble et al. 2005).

\section{Data acquisition and analysis}

For each arm and target amplitude, two trials were completed under a pseudo-randomized block design. Elbow position was recorded using precision potentiometers mounted beneath the pivot point of the manipulanda. The analog signal was digitized at $100 \mathrm{~Hz}$ and filtered (fourth-order Butterworth, zero phase lag, $6 \mathrm{~Hz}$ ) prior to data analysis. Three dependent measures were utilized in this study. Absolute error was defined as the absolute value of the difference between the target and matching movement end positions, where end position was calculated as the first point where baseline (zero) velocity was maintained $\pm 2 \mathrm{SD}$ for at least $1 \mathrm{~s}$. Second, movement smoothness was determined as the number of peaks in the velocity profile, where velocity peaks were determined based on the number of zero crossings in the acceleration profile divided by 2 . Lastly, average movement speed was calculated as the total movement time divided by total arm displacement where movement offset and onset were determined based on a deviation from baseline (zero) velocity $\pm 2 \mathrm{SD}$ and total displacement was quantified as the difference between the position values associated with movement onset and offset.

Given that an initial comparison of male versus female subject provided no indication of any gender differences in matching performance, statistical analysis consisted of a series of $2 \times 2$ ANOVAs with repeated measures for the main effects of matching arm (nonpreferred vs. preferred) and target position $\left(20^{\circ}\right.$ vs. $\left.40^{\circ}\right)$ as well as any interaction between these independent variables. Post hoc analyses were made using paired Student $t$ tests with statistical significance set at the level of $P \leq 0.01$ to compensate for any effects of multiple comparisons.

\section{Results}

Matching accuracy

Matching accuracy, as measured by absolute error, was significantly greater for the non-preferred compared to 


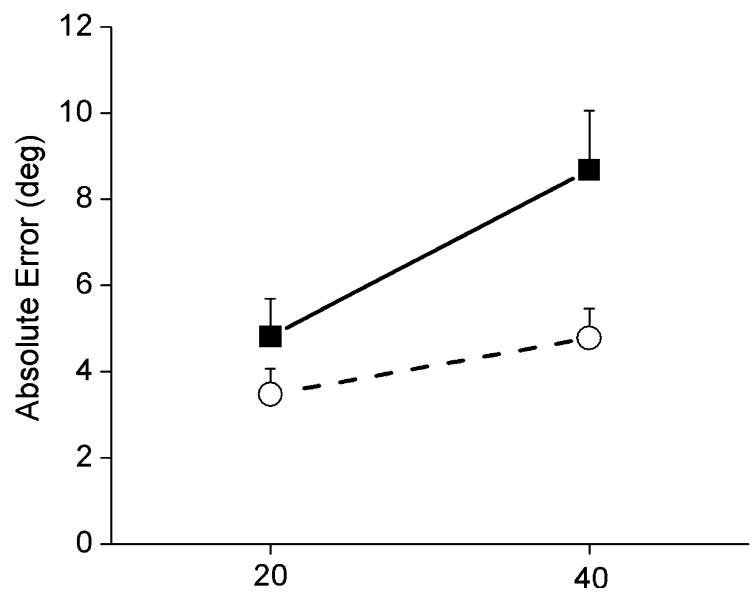

Fig. 1 Mean $( \pm$ SEM) differences in absolute error for matches made by the preferred (filled squares) and non-preferred (open circles) arms when matching $20^{\circ}$ and $40^{\circ}$ target positions

the preferred arm regardless of target position $\left(F_{(1,19)}=7.5, P<0.01\right)$. This non-preferred arm advantage is demonstrated in Fig. 1 where the average absolute error produced by the non-preferred arm was $40 \%$ less than that of the preferred arm. The effect of target position is also shown in Fig. 1 where a significant difference in matching error was seen for only the preferred arm with a near doubling of error in the $40^{\circ}$ as compared to the $20^{\circ}$ condition $\left(t_{(19)}=3.2, P<0.01\right)$.
Matching dynamics

Velocity profiles associated with proprioceptive matching were highly irregular as shown for a representative subject in Fig. 2a. Overall, these irregularities were more prominent in large compared to small amplitude movements (Fig. $2 \mathrm{~b} ; F_{(1,19)}=28.8, P<0.001$ ), although no significant differences in movement smoothness between the preferred and non-preferred arms were observed. Average movement speed increased with increasing movement amplitude regardless of which arm produced the matching task (Fig. $2 \mathrm{c} ; F_{(1,19)}=51.2, P<0.001$ ).

\section{Discussion}

The present study sought to determine the extent to which $\mathrm{arm} / \mathrm{hemisphere} \mathrm{differences} \mathrm{exist} \mathrm{in} \mathrm{the} \mathrm{utilization}$ of proprioceptive feedback. Overall, a non-preferred arm advantage was seen in the ability to actively reproduce passively determined target positions based on proprioceptive feedback. This advantage was most pronounced for larger amplitude movements and was not associated with any limb-specific difference in movement strategy as indicated by matching movement dynamics.

The fact that the non-preferred arm/hemisphere system was superior in its ability to match proprioceptive targets agrees with previous reports of a non-preferred
Fig. 2 a Representative velocity profiles associated with matching movements made by the preferred and non-preferred arms in the $20^{\circ}$ and $40^{\circ}$ target conditions. b Mean ( \pm SEM) movement smoothness for preferred (hatched) and nonpreferred (open) arm matchings in $20^{\circ}$ and $40^{\circ}$ target conditions. c Mean $( \pm$ SEM) average movement speed for preferred (hatched) and non-preferred (open) arm matchings in the $20^{\circ}$ and $40^{\circ}$ target conditions a
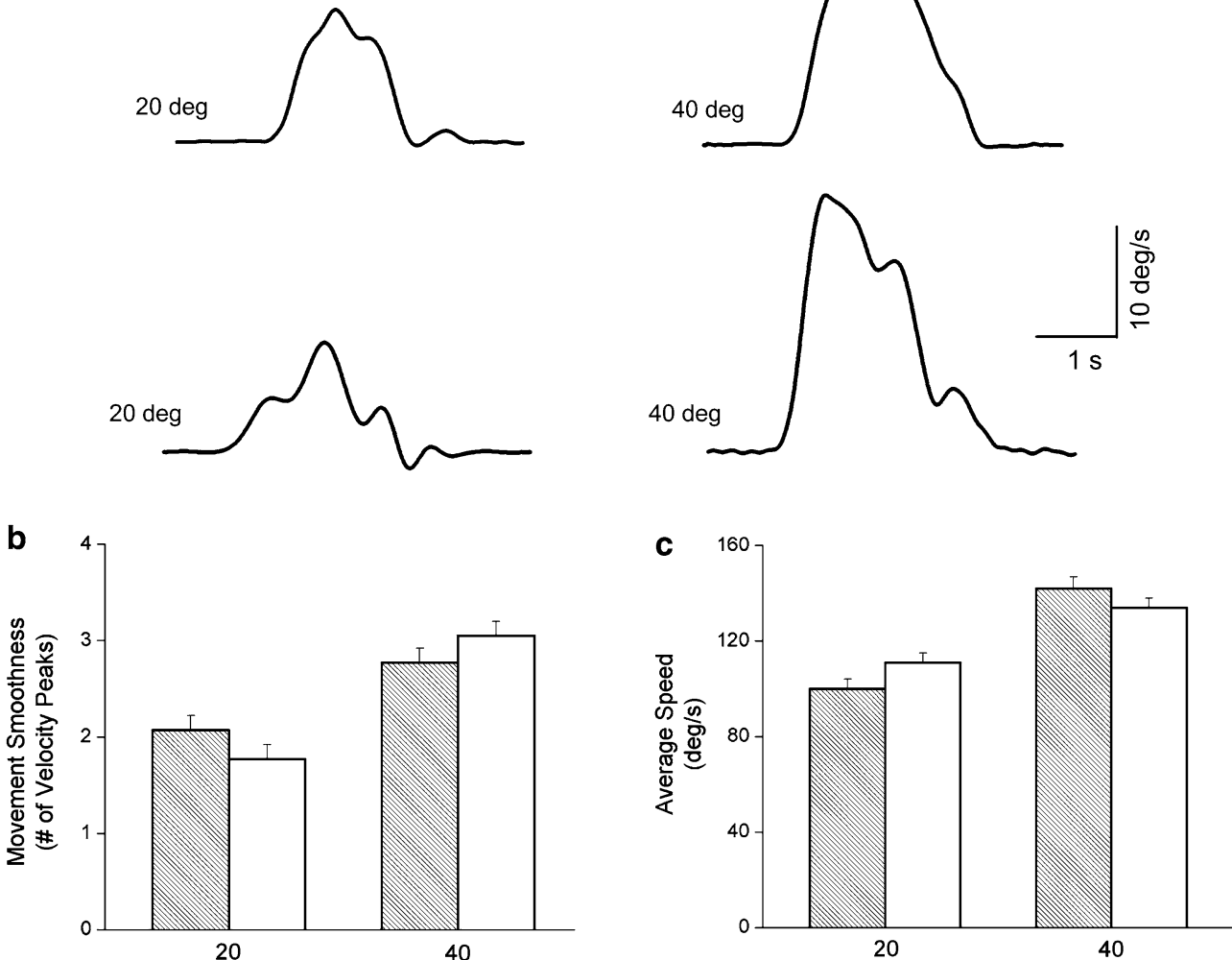
limb accuracy advantage for the thumb (Roy and MacKenzie 1978; Colley 1984) and foot (Carnahan and Elliott 1987). These findings are contrasted, however, by several studies that failed to show any limb asymmetry during proprioceptive matching (Roy and MacKenzie 1978; Carson et al. 1990; Chapman et al. 2001). This apparent lack of limb asymmetry during proprioceptive matching may be, to a large extent, explained by limitations in methodology. In studies where the target position was generated actively by the subject (Roy and MacKenzie 1978; Carson et al.1990), it is possible that, in addition to proprioceptive feedback, limb position information arising from efference during establishment of the target position may have influenced matching accuracy. Efferent copy mechanisms are thought to contribute to the generation of internal models of movement (for review see Kawato 1999) which, in the case of a proprioceptive matching paradigm, could enhance matching performance of the preferred limb. The notion that active generation of target position may provide movement-related information independent of proprioceptive feedback is supported by neuroimaging studies in which neural activation patterns associated with passive and active limb movements have been examined. For example, Mima et al. (1999) found that during passive movements, somatosensory cortex was primarily activated while, in contrast, active displacement of the limb resulted in a more distributed network of brain areas including somatosensory cortex, premotor cortex, supplementary motor area, basal ganglia and ipsilateral cerebellum.

Chapman et al. (2001) also reported a lack of limb asymmetries in proprioceptive matching during a task which, like the present study, utilized passive targets. In this case, it is possible that the demands of the task were not great enough to elicit significant limb differences. In the Chapman study a forward-reaching paradigm was utilized where memory-based movements were made to targets with the ipsilateral limb. In the present study, a memory-based task was also used, but one that required transfer of a proprioceptive memory of limb position across hemispheres in order to match with the contralateral arm. Indeed, asymmetric transfer of learning has been shown for motor activities such as finger sequencing (Taylor and Heilman 1980) and, more specifically, for limb position information in favor of the non-preferred arm/hemisphere during visuomotor adaptation (Wang and Sainburg 2003, 2004) and load compensation (Bagesteiro and Sainburg 2003) tasks. In addition, recent findings from this laboratory have shown that taskspecific differences exist in the proprioceptively guided movements of children (Goble et al. 2005). In the Goble study three elbow position matching tasks of varying complexity were assessed involving memory-based matching with the ipsilateral limb, "online" matching of the ipsilateral limb with the contralateral limb maintained in the target position or memory-based matching with the contralateral limb as used in the present study. Only in this latter case where a proprioceptive memory of limb position had to be generated and transferred across hemispheres were arm differences evident, suggesting that the emergence of limb asymmetries may partly depend upon task complexity such that greater processing of sensory information is required.

Overall, these results extend current theories of handedness by demonstrating that, in right-handed individuals, the non-preferred arm/hemisphere system is more adept at utilizing position-related proprioceptive information than the preferred arm/hemisphere system. Such an advantage may reflect a preferential role for the non-preferred arm/hemisphere system in the maintenance of static postures which might be necessary during, for example, bimanual tasks requiring object stabilization with one hand and manipulation with the other.

Acknowledgements Special thanks to J. Chesney for his assistance with the data collection and analysis aspects of this project.

\section{References}

Annett J, Annett M, Hudson PT, Turner A (1979) The control of movement in the preferred and non-preferred hands. Q J Exp Psychol 31:641-652

Bagesteiro LB, Sainburg RL (2003) Nondominant arm advantages in load compensation during rapid elbow joint movements. $\mathbf{J}$ Neurophysiol 90:1503-1513

Carnahan H, Elliott D (1987) Pedal asymmetry in the reproduction of spatial locations. Cortex 23:157-159

Carson RG, Elliott D, Goodman D, Dickinson J (1990) Manual asymmetries in the reproduction of a 3-dimensional spatial location. Neuropsychologia 28:99-103

Carson RG, Goodman D, Chua R, Elliott D (1993) Asymmetries in the regulation of visually guided aiming. J Mot Behav 25:2132

Chapman CD, Heath MD, Westwood DA, Roy EA (2001) Memory for kinesthetically defined target location: evidence for manual asymmetries. Brain Cogn 46:62-66

Colley A (1984) Spatial location judgements by right and lefthanders. Cortex 20:47-53

Cordo P, Carlton L, Bevan L, Carlton M, Kerr GK (1994) Proprioceptive coordination of movement sequences: role of velocity and position information. J Neurophysiol 71:18481861

Elliott D, Weeks DJ, Jones R (1986) Lateral asymmetries in fingertapping by adolescents and young adults with Down syndrome. Am J Ment Defic 90:472-475

Elliott D, Heath M, Binsted G, Ricker KL, Roy EA, Chua R (1999) Goal-directed aiming: correcting a force-specification error with the right and left hands. $\mathbf{J}$ Mot Behav 31:309-324

Flowers K (1975) Handedness and controlled movement. Br J Psychol 66:39-52

Goble DJ, Lewis CA, Hurvitz EA, Brown SH (2005) Development of upper limb proprioceptive accuracy in children and adolescents. Hum Mov Sci 24:155-170

Haaland KY, Harrington D (1989a) The role of the hemispheres in closed loop movements. Brain Cogn 9:158-180

Haaland KY, Harrington DL (1989b) Hemispheric control of the initial and corrective components of aiming movements. Neuropsychologia 27:961-969

Kawato M (1999) Internal models for motor control and trajectory planning. Curr Opin Neurobiol 9:718-727

Lonn J, Crenshaw AG, Djupsjobacka M, Pedersen J, Johansson H (2000) Position sense testing: influence of starting position and type of displacement. Arch Phys Med Rehabil 81:592-597 
Mieschke PE, Elliott D, Helsen WF, Carson RG, Coull JA (2001) Manual asymmetries in the preparation and control of goaldirected movements. Brain Cogn 45:129-140

Mima T, Sadato N, Yazawa S, Hanakawa T, Fukuyama H, Yonekura Y, Shibasaki H (1999) Brain structures related to active and passive finger movements in man. Brain $122(\mathrm{Pt}$ 10):1989-1997

Oldfield RC (1971) The assessment and analysis of handedness: the Edinburgh inventory. Neuropsychologia 9:97-113

Roy EA, MacKenzie C (1978) Handedness effects in kinesthetic spatial location judgements. Cortex 14:250-258

Roy EA, Kalbfleisch L, Elliott D (1994) Kinematic analyses of manual asymmetries in visual aiming movements. Brain Cogn $24: 289-295$
Sainburg RL (2002) Evidence for a dynamic-dominance hypothesis of handedness. Exp Brain Res 142:241-258

Sainburg RL, Ghilardi MF, Poizner H, Ghez C (1995) Control of limb dynamics in normal subjects and patients without proprioception. J Neurophysiol 73:820-835

Taylor HG, Heilman KM (1980) Left-hemisphere motor dominance in righthanders. Cortex 16:587-603

Wang J, Sainburg RL (2003) Mechanisms underlying interlimb transfer of visuomotor rotations. Exp Brain Res 149:520-526

Wang J, Sainburg RL (2004) Limitations in interlimb transfer of visuomotor rotations. Exp Brain Res 155:1-8

Winstein CJ, Pohl PS (1995) Effects of unilateral brain damage on the control of goal-directed hand movements. Exp Brain Res 105:163-174 